\title{
Avaliação dietética de amidos pelas respostas glicêmica e insulinêmica em cães
}

[Dietary evaluation of starches through glycemic and insulinemic responses in healthy dogs]

\author{
G.B. Silveira, B.P. Paule, E.P. Socorro
}

Escola de Medicina Veterinária da Universidade Federal da Bahia

Av. Ademar de Barros, 500 - Ondina

40110-170 - Salvador, BA

\begin{abstract}
RESUMO
Avaliaram-se, em cães saudáveis, os efeitos da fonte e do tipo de processamento do amido presente no alimento sobre as concentrações de insulina e glicose plasmáticas pós-prandiais. Foram utilizados oito cães da raça Beagle, adultos, alimentados com quatro regimes: amido cru de trigo (ACT), amido gelatinizado de trigo (AGT), amido cru de mandioca (ACM) e amido gelatinizado de mandioca (AGM). As amostras de sangue foram coletadas mediante punção da veia braquial aos $0,70,140,250$ e 360 minutos pós-prandiais. O regime não influenciou a glicemia, mas alterou a concentração sangüínea de insulina $(\mathrm{P}<0,05)$. Na área total sob a curva insulinêmica, o regime ACT não diferiu do AGM e foi superior aos regimes AGT e ACM, em razão da viscosidade do AGT e da resistência ao ataque enzimático do ACM. Os resultados sugerem que o regime AGT deve ser preferido aos demais por minimizar a resposta insulínica pós-prandial e manter a euglicemia.
\end{abstract}

Palavras-chave: cão, amido, glicemia, insulinemia

\begin{abstract}
The effects of both starch source and processing in dog chow on postprandial plasma glucose and insulin concentrations in eight healthy adult Beagle dogs fed on four dietary regimens, raw wheat starch (RWS), gelatinized wheat starch (GWS), raw cassava starch (RCS) and gelatinized cassava starch (GCS) were studied. Blood samples were collected by brachial vein puncture at 0, 70, 140, 250 and 360 minutes postprandially. The diet had no influence on glycemia, but changed serum insulin concentration $(P<0.05)$. Total areas under the insulinemic curve did not differ for RWS and GCS diets, but they were larger than those for GWS and RCS, as a result of the viscosity of GWS and of the resistance to enzymatic attack of RCS diet. These results suggest that GWS diet could be indicated to minimize the insulinic postprandial response and to maintain the euglycemia.
\end{abstract}

Keywords: dog, starch, glycemia, insulinemia

\section{INTRODUÇÃO}

A alimentação de cães é um fator de crescente importância na atualidade, com largo potencial de crescimento. O mercado brasileiro de rações para cães, especialmente o nordestino, apresenta os maiores índices de crescimento mundial, tornando desejável a utilização de matériasprimas tropicais para reduzir o custo da ração. Os carboidratos digestíveis fornecem calorias mais baratas, representando elevado percentual na maior parte dos alimentos para animais de companhia. A introdução de amido na dieta acarreta uma economia notável de proteínas, que poderão ser reservadas à proteossíntese.

A natureza diversa dos amidos acarreta grandes diferenças na digestão e influi na eficácia metabólica e na saúde do animal. O tipo de alimento, seu processamento e o quanto isso

Recebido para publicação em 12 de maio de 2003

Recebido para publicação, após modificações, em 17 de agosto de 2004

*Autor para correspondência

E-mail: eliomar@ufba.br 
reflete na sua forma físico-química levam a diferentes influências nas respostas glicêmica e insulinêmica aos carboidratos dietéticos (Holm et al., 1988; Holste et al., 1989; Tovar et al., 1992; Muir et al., 1995; Nguyen et al., 1998a).

Uma boa avaliação das fontes amiláceas pode ser feita a partir do impacto provocado por um alimento nas respostas glicêmica e insulinêmica do animal, esclarecendo a utilização pelo organismo (Holste et al., 1989; Nguyen et al., 1994).

Este trabalho objetivou avaliar duas fontes de carboidratos, uma tradicional (trigo) e uma alternativa (mandioca), pela comparação do efeito dos seus amidos nos estados cru e gelatinizado sobre a resposta glicêmica e insulinêmica de cães.

\section{MATERIAL E MÉTODOS}

Foram utilizados oito cães (três machos e cinco fêmeas) da raça Beagle, adultos, de peso médio igual a $14,3 \pm 2,5 \mathrm{~kg}$. Todos os animais possuíam registro, tatuagem e foram submetidos a exames clínicos complementados por análises laboratoriais de rotina (hemograma e sumário de urina). Os cães estavam devidamente imunizados contra hepatite, cinomose, leptospirose e raiva, e 15 dias antes do início do experimento foram vermifugados. Acetato de medroxiprogesterona $(5 \mathrm{mg} / \mathrm{kg}$ de peso vivo) foi administrado às fêmeas para impedir o aparecimento do cio durante a pesquisa. Os cães foram pesados no início e no final de cada período do experimento.

Quatro regimes foram elaborados a partir de uma base protéica, em conserva, exclusivamente de origem animal, de modo a cobrir as necessidades de proteínas dos cães, fixadas em 4,8 gramas de proteína bruta por quilo de peso vivo (Nutrient..., 1985).

Cada regime alimentar foi definido pelo tipo de amido adicionado: amido cru de trigo (ACT), amido gelatinizado de trigo (AGT), amido cru de mandioca (ACM) e amido gelatinizado de mandioca (AGM).

Os regimes foram oferecidos aos cães uma única vez ao dia, pela manhã, durante 10 dias. A quantidade de amido puro adicionado em cada dieta foi calculada em base de matéria seca para obtenção de um regime que fornecesse $22 \mathrm{~g}$ de matéria seca por quilo de peso vivo. O objetivo de se levar em conta a matéria seca de cada amido foi o de permitir que todas as variações das características estudadas fossem exclusivamente atribuídas ao amido adicionado à ração.

Em termos de macroingredientes, os regimes estudados foram, segundo sua composição, semelhantes às rações encontradas no comércio. A única diferença baseou-se na substituição da farinha de cereais como fonte de carboidratos por amido puro, ou na utilização do amido de tubérculo no caso dos regimes ACM e AGM (Tab. 1).

Tabela 1. Composição química dos regimes oferecidos aos cães com base na matéria seca (média e desvio-padrão)

\begin{tabular}{lcccccc}
\hline Regime & MSTA & MMAS & MOAS & AMAS & PBAS & EBAS \\
\hline \multirow{2}{*}{ ACT } & 34,85 & 6,64 & 93,12 & 48,82 & 25,88 & 4766,90 \\
& $(0,32)$ & $(0,08)$ & $(0,08)$ & $(2,30)$ & $(1,68)$ & $(37,92)$ \\
\multirow{2}{*}{ AGT } & 34,85 & 7,14 & 92,86 & 49,35 & 25,51 & 4789,13 \\
& $(0,28)$ & $(0,17)$ & $(0,17)$ & $(1,26)$ & $(1,45)$ & $(22,26)$ \\
\multirow{2}{*}{ ACM } & 34,76 & 6,97 & 93,03 & 50,73 & 24,69 & 4739,27 \\
& $(0,64)$ & $(0,12)$ & $(0,12)$ & $(1,77)$ & $(2,03)$ & $(58,17)$ \\
\multirow{2}{*}{ AGM } & 34,57 & 6,77 & 93,23 & 50,37 & 24,85 & 4689,20 \\
& $(0,43)$ & $(0,24)$ & $(0,24)$ & $(2,04)$ & $(1,00)$ & $(59,92)$ \\
\hline
\end{tabular}

Fonte: Wolter et al. (1998). MSTA - matéria seca total do alimento; MMAS - matéria mineral do alimento seco; MOAS - matéria orgânica do alimento seco; AMAS - amido do alimento seco; PBAS - proteína bruta do alimento seco; EBAS - energia bruta do alimento seco. 
Os três regimes foram classificados como alimentos do tipo semi-úmido, apesar de sua composição em proteína bruta, carboidratos e minerais ser muito mais próxima dos alimentos secos encontrados no mercado (Tab. 1).

Nas últimas 72 horas do período de adaptação e durante o experimento, os cães foram alojados em gaiolas individuais de modelo clássico, segundo as normas de detenção de animais de laboratório da CCE-Diretiva Européia 86/609/CCE. A temperatura do galpão do experimento foi controlada entre 16 e $21^{\circ} \mathrm{C}$.

Para cada alimento colheu-se uma subamostra de 40 gramas no momento da sua distribuição aos cães. A pré-secagem foi feita imediatamente à $65^{\circ} \mathrm{C}$ sob ventilação, até se obter umidade inferior a $12 \%$. Após o resfriamento à temperatura ambiente, pesava-se novamente a subamostra para a determinação individual da matéria seca ao ar, estocando-a em câmara fria $\left(5^{\circ} \mathrm{C}\right)$.

No fim de cada período experimental, o conjunto das subamostras era moído ${ }^{1}$ para que fossem obtidas partículas com tamanho médio de $1 \mathrm{~mm}$. Em seguida as subamostras eram reagrupadas e homogeneizadas, formando amostra única para cada alimento e período, a partir das quais calculava-se o valor médio do teor de matéria seca ao ar.

Para a determinação da glicose e insulina, as amostras de sangue foram colhidas imediatamente antes do início da ingestão dos regimes (tempo 0) e aos 70, 140, 250 e 360 minutos após a ingestão da dieta. Todas as coletas foram feitas por punção da veia braquial. $\mathrm{O}$ sangue, imediatamente centrifugado a $600 \mathrm{x} \mathrm{g}$ por 15 minutos para separação do plasma, foi utilizado para análise de glicose por kit enzimático ${ }^{2}$ e estocado $\left(-20^{\circ} \mathrm{C}\right)$ até o momento da análise de insulina por radioimunoensaio.

\footnotetext{
${ }^{1}$ Moinho Edmund Bühler Laborgerätebeau, tipo Glastechnik

Unwelttechnick

${ }^{2}$ Ames Division, Miles Laboratories, Inc. Co.
}

O delineamento experimental utilizado foi o de dois quadrados latinos independentes de dimensão $4 \times 4$, com distribuição inteiramente ao acaso dos cães por quadrado latino. A justificativa da escolha desse delineamento repousa sobre o número de repetições (cães) necessárias no ensaio em relação ao número de gaiolas de metabolismo disponíveis. Desse modo, quando quatro cães estavam em período experimental (quadrado latino 1), quatro cães estavam em período de adaptação (quadrado latino 2). Cada período teve a duração de 10 dias.

O estudo estatístico foi feito a partir da comparação das áreas (AutoCad, 2000) sob as curvas de glicose e insulina sangüíneas segundo o regime alimentar em função do tempo. O consumo foi usado como covariável, e as médias foram comparadas pelo teste $\mathrm{t}$ de Student (Sampaio, 1998).

\section{RESULTADOS E DISCUSSÃO}

Os valores médios de glicose e insulina sangüíneas de acordo com o regime alimentar e o tempo de avaliação são, respectivamente, apresentados nas Fig. 1 e 2. Para que as respostas aos diferentes regimes pudessem ser analisadas estatisticamente pela comparação gráfica nos diferentes intervalos de tempo, foram medidas as áreas de superfície sob a curva, utilizando-se a mesma escala por tempo e por regime. Tais medidas permitiram conhecer a amplitude das respostas, evidenciando as diferenças entre os regimes.

Verificou-se similaridade nos valores de concentração da glicose segundo o regime alimentar. Essa tendência também foi encontrada por Nguyen et al. (1994). Nenhum dos regimes pode ser considerado hiperglicemiante, uma vez que as médias de concentração sangüínea da glicose não ultrapassaram o limite de $130 \mathrm{mg} / \mathrm{dl}$ citado por Holste et al. (1989). 


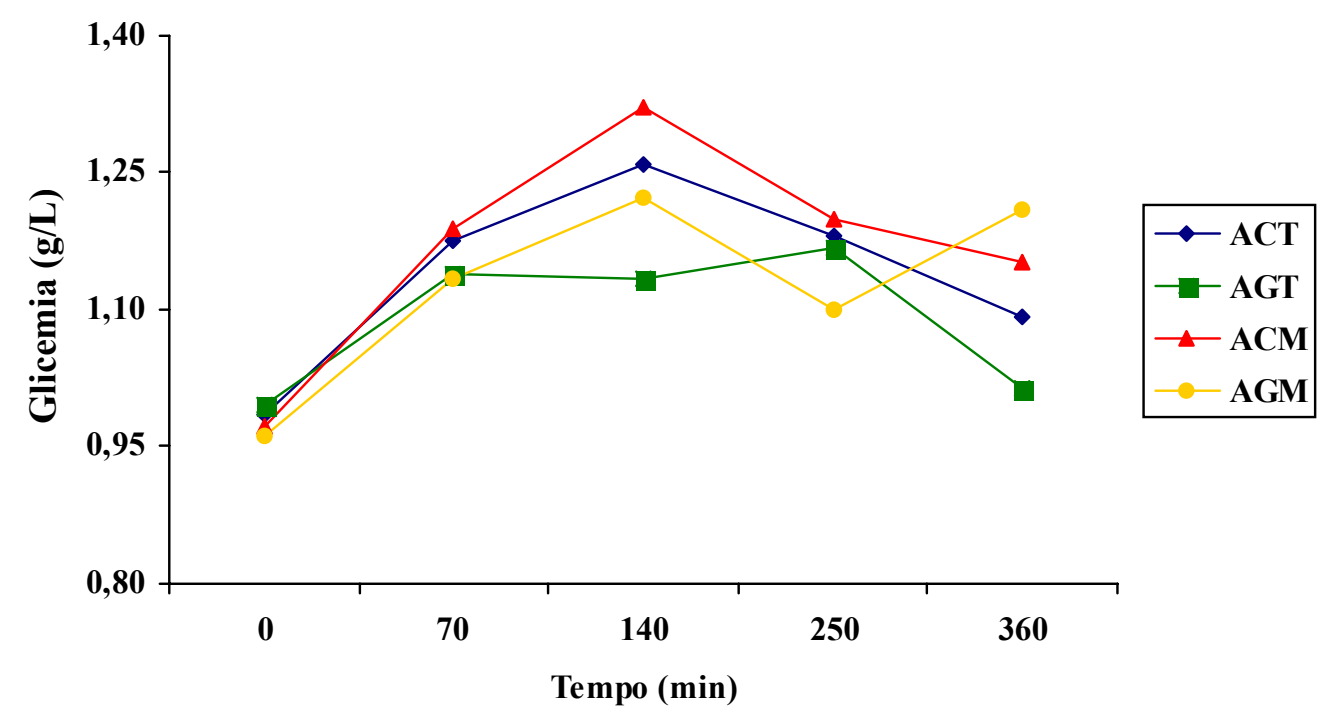

Figura 1. Variações na glicemia segundo o tempo pós-prandial e o tratamento.

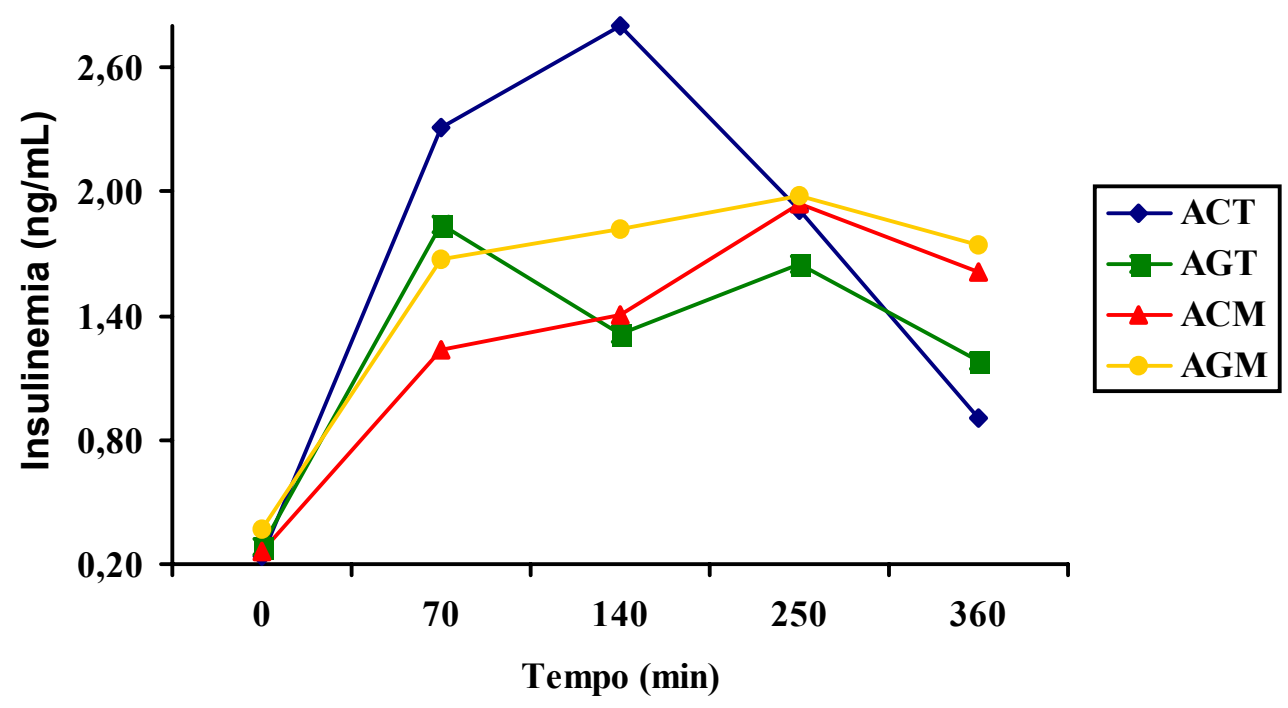

Figura 2. Variações na insulinemia segundo o tempo pós-prandial e o tratamento. 
O pico de glicemia ocorreu no intervalo entre 140 e 250 minutos pós-prandiais para todos os tratamentos (Fig. 1). A primeira elevação da glicemia, uniforme, ocorreu aos 70 minutos, comum aos quatro regimes estudados. Diferentemente dos demais, o AGT apresentou pequena redução na glicemia logo após seu primeiro pico, que pode ser interpretada como uma resposta à primeira elevação da insulinemia nesse mesmo tempo (Perley e Kipnis, 1967). A segunda elevação da insulina sangüínea poderia estar ligada à liberação lenta, porém contínua, da glicose nos intestinos, relacionada com a viscosidade do regime (Leport e Clerf, 1987). A baixa resposta insulinêmica registrada com o regime AGT seria, dessa maneira, a conseqüência direta desse fenômeno, uma vez que qualquer processo que rompa a estrutura física ou botânica dos ingredientes do alimento aumenta o tempo de resposta glicêmica e insulinêmica. Assim, o grau final de gelatinização pode ser um fator que determina o índice de digestão do amido e a resposta glicêmica subseqüente, pela destruição da estrutura dos grânulos de amido, aumentando a susceptibilidade à degradação por alfamilase (Nguyen et al., 1998a). As dietas à base de amidos crus (ACT e ACM) apresentaram picos de concentração de glicose sangüínea aos 140 minutos. No entanto, o AGM, apesar de ter apresentado seu teor máximo de glicose também aos 140 minutos, apresentou nova elevação aos 360 minutos, mesma propensão quanto à formação de dois piques do regime AGT, o qual teve a segunda elevação aos 250 minutos, levemente mais alta que a primeira aos 70 minutos.

O tipo de regime repercutiu sobre a concentração sangüínea de insulina $(\mathrm{P}<0,05)$. Na área total sob a curva insulinêmica, o regime ACT não diferiu estatisticamente do AGM e foi superior aos regimes AGT e ACM. A intensidade da resposta insulinêmica descrita pelas curvas dos regimes ACT e AGM é típica dos alimentos ricos em glicídios solúveis, ou seja, dos alimentos semiúmidos (Holste et al., 1989). No entanto, a aparição do pico insulinêmico desses dois regimes no intervalo de tempo de 140 a 250 minutos corresponde mais àquele dos alimentos contendo amidos (alimentos secos e úmidos), semelhante ao tempo 30 minutos observado por Holste et al. (1989) com alimentos contendo açúcares simples (alimentos semi-úmidos).
Tendência similar na concentração de insulina plasmática para os quatro regimes foi observada no intervalo entre 140 e 250 minutos. Esse intervalo também coincidiu com a máxima concentração de insulina plasmática, com exceção do regime $\mathrm{ACM}$, que atingiu seu pico de concentração no intervalo de 250 a 360 minutos. Nesse tempo não houve diferença significativa entre os regimes $(\mathrm{P}>0,05)$, assim como no intervalo de 0 a 70 minutos. No intervalo de tempo entre 70 e 140 minutos pós-prandiais, o regime ACT foi diferente dos demais $(\mathrm{P}<0,05)$, apresentando a maior concentração sangüínea de insulina. Os AGM e ACT apresentam um traçado de curva insulinêmica similar em termos de amplitude de resposta e intervalo de aparição do pico, o que indica utilização equivalente desses amidos pelos cães, fato confirmado pelos resultados de digestibilidade ileal desses mesmos regimes (Wolter et al., 1998).

O comportamento das curvas glicêmica e insulinêmica em relação aos diferentes regimes estão de acordo com as constatações de Nguyen et al. (1998b) quanto à diminuição da glicose sangüínea em resposta à liberação insulínica estimulada por carboidratos rapidamente absorvíveis. Essa diminuição poderia, ainda, induzir a secreção de hormônios contraregulatórios (glucagon, hormônio de crescimento ou cortisol) e a liberação de ácidos graxos que, conseqüentemente, influenciariam as concentrações glicêmicas e insulinêmicas. As respostas obtidas nos diversos tempos de coleta sangüínea discordam dos achados desses autores. Eles afirmaram que uma amostragem sangüínea por um período superior a 90 minutos seria desnecessária para caracterizar as respostas glicêmica e insulinêmica em cães saudáveis. Nesse caso, este trabalho não detectaria o segundo pico glicêmico do amido gelatinizado de trigo, aos 250 minutos, e do amido gelatinizado de mandioca, aos 360 minutos. O nível insulinêmico basal não foi restabelecido durante as seis horas estudadas para nenhum dos regimes. Isto volta a conflitar com as constatações de Holste et al. (1989) para o cão porque, apesar das respostas insulinêmicas nos tempos 70 e 140 minutos terem sido capazes de indicar diferença entre os amidos, não mostraram o comportamento completo, o que é verdadeiramente importante para avaliar os possíveis efeitos na saúde do animal. Dessa maneira, maior número de coletas sangüíneas 
permitiram conhecer melhor os efeitos dos amidos estudados sobre as respostas glicêmica e insulinêmica.

Para o regime AGM, parece que a primeira elevação da glicemia teria sido freada somente pela elevação simultânea de insulina, e que a quantidade secretada de insulina não teria sido capaz de conter a carga total de glicose que chega até 360 minutos.

No caso do regime ACM, a contradição constatada entre a baixa digestibilidade ileal do amido (Wolter et al., 1998) e a glicemia relativamente forte, acompanhada de baixa insulinemia, mostraria um efeito particular do amido cru da mandioca sobre a resposta insulinêmica (Kamalu, 1991). Esse efeito teria desaparecido com a gelatinização.

Diferentemente das observações feitas por Holm et al. (1988) no rato, o retorno aos valores da glicose em jejum não permite diferenciar amidos crus e gelatinizados. Em compensação, os resultados obtidos discriminariam a origem botânica dos amidos, uma vez que o retorno aos valores glicêmicos basais não foi atingido no intervalo de tempo estudado com os regimes à base de amido de mandioca.

Não obstante a ausência de diferenças estatísticas entre os regimes estudados quanto à glicemia, as concentrações sangüíneas da insulina foram diferentes entre os regimes. A ordem de grandeza das respostas insulinêmicas entre regimes não corresponde àquela da glicemia, tendência essa não observada por Nguyen et al. (1998b), os quais encontraram correlação entre os valores de glicose e insulina sangüíneas. Os resultados apresentados confirmam às observações de Chen e Porte (1976), as quais indicam que o pico da insulina não depende somente da quantidade de glicose que chega no sangue, mas também da velocidade de seu fluxo e da relação entre elas. Nguyen et al. (1998a) também não encontraram correlação entre as áreas sob as curvas de glicose e insulina e sugerem que em cães clinicamente saudáveis o conteúdo de amido é o determinante primário da glicemia pós-prandial, enquanto que os conteúdo de proteína, estrato etéreo e amido parecem determinar a resposta insulínica.

As diferenças estatísticas entre os regimes foram colocadas em evidência pela comparação das taxas médias de glicose e de insulina sangüíneas, e também pela comparação das superfícies sob as curvas de seus gráficos. Essas superfícies representam a carga total da glicose liberada durante o tempo estudado, obedecendo a relação quantidade/tempo, de acordo com as observações de Lerner e Porte (1971), Olefsky et al. (1977), Rottiers et al. (1981) e Nguyen et al. (1998b).

Outro fator que pode ter tido um papel marcante na similaridade da intensidade da resposta glicêmica dos regimes é a clearance renal da glicose sangüínea, descrita por Kaneko et al. (1978), que acontece durante os intervalos de tempo que separam os horários de coleta das amostras sangüíneas.

Enfim, embora tenham sido controladas algumas variáveis como estresse, composição da dietabase e quantidade de amido, em base de matéria seca, todas as variações da glicemia e da insulinemia, no caso do presente estudo terão certamente diferentes explicações, em razão do grande número de fatores que interferem nestes resultados, tais como estrutura físico-química, tamanho das partículas, processamento, digestibilidade e grau de viscosidade (Leport e Clerf, 1987; Tovar et al., 1992; Holt e Miller, 1994; Muir et al., 1995), de tal modo que o efeito final sobre a curva da glicemia pós-prandial seja um reflexo do conjunto de fatores inerentes aos amidos adicionados ao regime.

Informações sobre o comportamento glicêmico e insulinêmico pós-prandiais podem ser de grande interesse no manejo de diabetes melito não insulinodependente (o qual cursa com alteração da tolerância aos carboidratos e ação insulínica), bem como nas diabetes melito insulinodependente, pela redução na flutuação glicêmica e sincronização do aumento da glicose com a administração de insulina (Nguyen et al., 1994).

A partir dos resultados deste trabalho, o perfil pós-prandial da insulinemia evidenciou diferenças entre os quatro regimes estudados. A resposta insulinêmica dos regimes AGT e ACM foram menos pronunciadas que os demais regimes. Esse comportamento pode ser relacionado com a viscosidade do regime AGT e com a resistência ao ataque enzimático do ACM. Estes resultados sugerem que o regime AGT, nas condições estudadas, deve ser preferido aos 
demais por minimizar a resposta insulínica pósprandial e manter a euglicemia. Essas conclusões podem ser de grande interesse para o manejo dietético de algumas doenças nutricionais comuns em cães, tal como obesidade e diabetes.

\section{AGRADECIMENTOS}

Ao professor R. Wolter (in memoriam) pelo apoio no desenvolvimento do experimento na Escola Nacional de Veterinária de Alfort (França).

\section{REFERÊNCIAS BIBLIOGRÁFICAS}

AUTO CAD 2000: autodesk software. San Rafael, CA: Autodesk, 2000.

CHEN, M.; PORTE, D. Jr. The effect of rate and dose of glucose infusion on the acute insulin response in man. Clin. Endocrinol. Metab., v.42, p.1168-1175, 1976.

HOLM, J.; LUNDQUIST, I.; BJÖRCK, I. et al. Degree of starch gelatinization, digestion rate of starch "in vitro" and metabolic response in rats. Am. J. Clin. Nutr., v.47, p.1010-1016, 1988.

HOLSTE, L.C.; NELSON, R.W.; FELDMAN, E.C. et al. Effect of dry, soft moist, and canned dog foods on postprandial blood glucose and insulin concentrations in healthy dogs. Am. J. Vet. Res., v.50, p.984-989, 1989.

HOLT, S.H.; MILLER, J.B. Particle size, satiety and glycaemic response. Eur. J. Clin. Nutr., v.48, p.496502, 1994.

KAMALU, B.P. The effect of a nutritionally-balanced cassava (Manihot esculenta Crantz) diet on endocrine function using the dog as a model. 1. Pancreas. Br. J. Nutr., v.65, p.365-372, 1991.

KANEKO, J.J.; MATTHEEUWS, D.; ROTTIERS, R.P. et al. The effect of urinary glucose excretion on the plasma glucose clearances and plasma insulin responses to intravenous glucose loads in unanaesthesized dogs. Acta Endocrinol., v.87, p.133138,1978 .

LEPORT, J.; CLERF, M. Digestion et digestibilité de l'amidon. Cah. Nutr. Digestib., v.22, p.435-441, 1987.
LERNER, R.L.; PORTE, D. Jr. Relationship between intravenous glucose loads, insulin response and glucose disappearence rate. J. Clin. Endocrinol. Metab., v.33, p.409-417, 1971.

MUIR, J.G.; BIRKETT, A.; BROWN, I. et al. Food processing and maize variety affects amounts of starch escaping digestion in the small intestine. Am. J. Clin. Nutr., v.61, p.82-89, 1995.

NGUYEN, P.; DUMON, H.; BIOURGE, V. et al. Glycemic and insulinemic responses after ingestion of commercial foods in healthy dogs: influence of food composition. J. Nutr., v.128, p.2654S-2658S, 1998 a.

NGUYEN, P.; DUMON, H.; BIOURGE, V. et al. Measurement of postprandial incremental glucose and insulin changes in healthy dogs: influence of food adaptation and length of time of blood sampling. $J$. Nutr., v.128, p.2659S-2662S, 1998b.

NGUYEN, P.; DUMON, H.; BUTTIN, P. et al. Composition of meal influences changes in postprandial increment at glucose and insulin in healthy dogs. J. Nutr., v.124, p.2707S-2711S, 1994.

NUTRIENT requirements of dogs. Washington: National Academy of Science, 1985.

OLEFSKY, J.M.; BARCHELDER, T.; FARQUHAR, J.W. Dissociation of plasma insuline response from the blood glucose concentration during glucose infusions in normal dogs. Metabolism, v.22, p.1277$1286,1977$.

PERLEY, M.J.; KIPNIS, D.M. Plasma insulin responses to oral and intravenous glucose: studies in normal and diabetic subjects. J. Clin. Invest., v.46, p.1954-1962, 1967.

ROTTIERS, R.; MATTHEEUWS, D.; KANEKO, J.J. Glucose uptake and insulin secretory responses to intravenous glucose loads in the dog. Am. J. Vet. Res., v.42, p.155-158, 1981.

SAMPAIO, I.B.M. Estatística aplicada à experimentação animal. Belo Horizonte: Fundação de Ensino e Pesquisa em Medicina Veterinária e Zootecnia, 1998.

TOVAR, J.; GRANFELDT, Y.; BJÖRCK, M. Effects of processing on blood glucose and insulin responses to starch in legumes. J. Agric. Food Chem., v.40, p.1846-1851, 1992.

WOLTER, R.; SOCORRO, E.P.; HOUDRÉ, C. Digestibilité totale et iléale d`aliments à haute teneur en amidon de blé ou de manioc chez le chien. Rec. Méd. Vét., v.174, p.45-55, 1998. 